Check for updates

Cite this: Chem. Commun., 2019, 55, 9535

Received 14th May 2019, Accepted 9th July 2019

DOI: $10.1039 /$ c9cc03719e

rsc.li/chemcomm

\section{Activity-based protein profiling reveals that secondary-carbon-centered radicals of synthetic 1,2,4-trioxolanes are predominately responsible for modification of protein targets in malaria parasites $\dagger$}

\author{
Chunyan Wei, $\ddagger^{a}$ Cheng-Xiao Zhao, $\ddagger^{\text {bc }}$ Sheng Liu, $\ddagger^{\mathrm{b}}$ Jin-Hui Zhao, $\ddagger^{\mathrm{b}} \mathrm{Zi}$ Ye, ${ }^{\mathrm{b}}$ \\ Heng Wang, ${ }^{\mathrm{ab}}$ Shi-Shan $\mathrm{Yu}^{{ }^{\mathrm{b}}}$ and Chong-Jing Zhang (D) ${ }^{\mathrm{b}}$
}

\begin{abstract}
Endoperoxide-containing antimalarials, such as artemisinin and the synthetic trioxolane OZ439, are prodrugs activated by heme to generate primary and secondary carbon-centered radicals. We employed activity-based protein profiling (ABPP) to show that the secondary-carbon-centered radical of 1,2,4-trioxolanes is primarily responsible for protein labeling in malaria parasites.
\end{abstract}

Malaria caused by the protozoal parasite Plasmodium falciparum and related species is a mosquito-borne and life-threating infectious disease, which leads to nearly a half million deaths globally each year. ${ }^{1}$ The first small molecule drug used to treat malaria was quinine, extracted from the cinchona tree. ${ }^{2}$ Due to limited access to this natural source during the Second World War, chloroquine, a synthetically-derived quinoline was identified as an anti-malaria drug. ${ }^{3}$ Chloroquine and its derivatives were extensively used from the 1930s until the 1950 s. ${ }^{4}$ Unfortunately, widespread resistance to chloroquine eventually developed, prompting a search for next generation anti-malaria drugs. Chinese scientists led by Youyou Tu discovered the fascinating natural product artemisinin (Qinghaosu) and showed that it possesses unparalleled efficacy for treatment of malaria including complicated malaria caused by chloroquine-resistant plasmodial strains. ${ }^{5}$ Artemisinin established the pivotal role of the endoperoxide as a unique and effective antimalarial pharmacophore. ${ }^{6}$ The

\footnotetext{
${ }^{a}$ Department of Microbiology and Parasitology, Institute of Basic Medical Sciences, Chinese Academy of Medical Sciences \& School of Basic Medicine, Peking Union Medical College, 5\# Dong Dan San Tiao, Beijing, 100005, China. E-mail: wanghpumc@163.com

${ }^{b}$ State Key Laboratory of Bioactive Substances and Functions of Natural Medicines and Beijing Key Laboratory of Active Substances Discovery and Drugability Evaluation, Institute of Materia Medica, Peking Union Medical College and Chinese Academy of Medical Sciences, Beijing, 100050, China. E-mail: yushishan@imm.ac.cn, zhangchongjing@imm.ac.cn ${ }^{c}$ School of Pharmaceutical Science, Shanxi Medical University, Taiyuan, Shanxi, 030001, China

$\dagger$ Electronic supplementary information (ESI) available: Intermediates and methods, and supplementary and extended figures. See DOI: 10.1039/c9cc03719e \$ These authors contributed equally to this work.
}

disadvantages of artemisinin are its rapid clearance in vivo, limited supply and impractical de novo synthesis. These challenges stimulated the development of synthetically-accessible and longer-acting trioxolanes, such as OZ277 and OZ439. ${ }^{7}$ Mechanism of action (MOA) studies revealed that the unique endoperoxide group in artemisinin and synthetic trioxolanes was first activated by heme to generate carbon-centered radicals, which subsequently alkylate multiple proteins in malaria parasites. ${ }^{8}$ During the activation of the endoperoxide by heme, two different radicals are generated, namely, primary and secondary carbon-centered radicals, each with their own unique properties (Fig. 1A and B). ${ }^{9}$ The primary carbon-centered radical is sterically smaller while the secondary-carbon-centered radical is more stable. ${ }^{10}$ However, the relative contributions of each radical for antimalarial activity and specific protein targets have not been elucidated. Herein we determine the protein targets of each radical and further refine

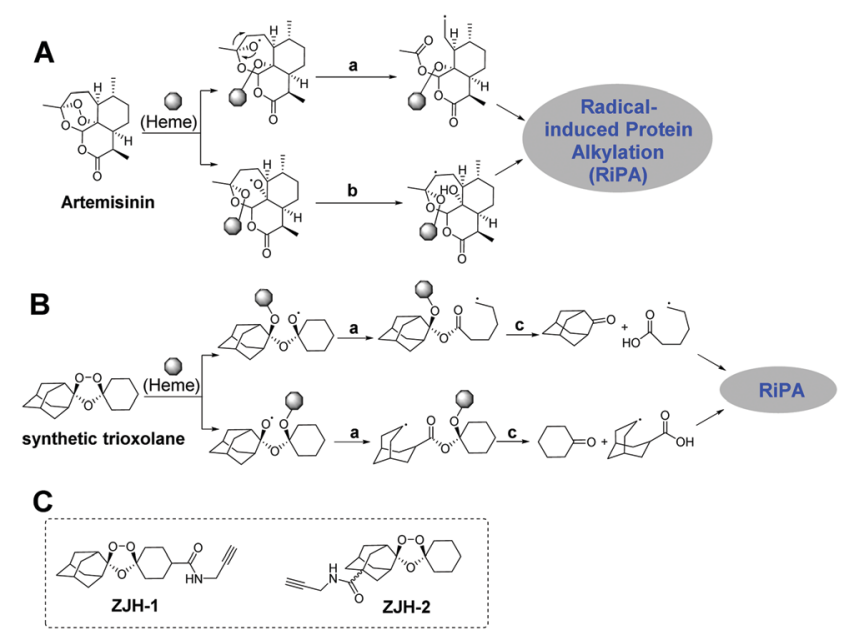

Fig. 1 Potential reaction pathways of artemisinin (A) and a synthetic trioxolane (B) activated by heme. (a) Rearrangement; (b) 1,5- $\mathrm{H}$ shift; (c) cleavage. (C) The structures of probes used in this study, $\mathrm{ZJH}-1$ and $\mathrm{ZJH}-2$. RiPA, radical-induced protein alkylation. 
our mechanistic understanding of endoperoxide-containing antimalaria molecules.

To answer the above-mentioned mechanistic questions, we used activity-based protein profiling (ABPP) to capture the alkylated proteins of each radical. This was accomplished by designing two chemical probes containing an alkyne group. Alkynes are typically used in ABPP because they are sterically small and can be selectively conjugated to a fluorophore or an affinity tag employing bioorthogonal click-chemistry. ${ }^{11}$ Artemisinin is not an ideal substrate for these studies since both the radicals remain in the same scaffold (Fig. 1A); thus it is impossible to discern the specific proteins labeled by each radical regardless of where the alkyne group is introduced. On the other hand, the synthetic trioxolane provides an ideal scaffold for our proposed investigation. After heme-mediated activation followed by rearrangement and hydrolysis, synthetic trioxolane is transformed into either an adamantane ketone and a primary-carbon-centered radical, or a cyclohexane ketone and a secondary-carbon-centered radical (Fig. 1B). The protein targets of the two radicals can be easily traced by selecting appropriate attachment sites for the alkyne group. Following this design principle, incorporation of the alkyne into the cyclohexane part (ZJH-1, Fig. 1C) will capture the protein targets of the primary-carbon-centered radical while attachment of the alkyne group into the adamantane part $(\mathrm{ZJH}-2$, Fig. 1C) will capture the protein targets of the secondary-carboncentered radical.

Following this rationale, we synthesized probes $\mathrm{ZJH}-1$ and ZJH-2 (Scheme 1). Oxime 1 and 2 were synthesized via the reaction between the corresponding ketone and methoxyamine. The Griesebaum co-ozonolysis of 4-(methoxycarbonyl)cyclohexanone with oxime 2 afforded trioxolane 3. Saponification of 3 followed by EDC-mediated coupling with propargyl amine provided the probe $\mathrm{ZJH}-1$. Probe $\mathrm{ZJH}-2$ was synthesized in a similar manner as described. ${ }^{8 c}$ The chemical structures of probes $\mathrm{ZJH}-1$
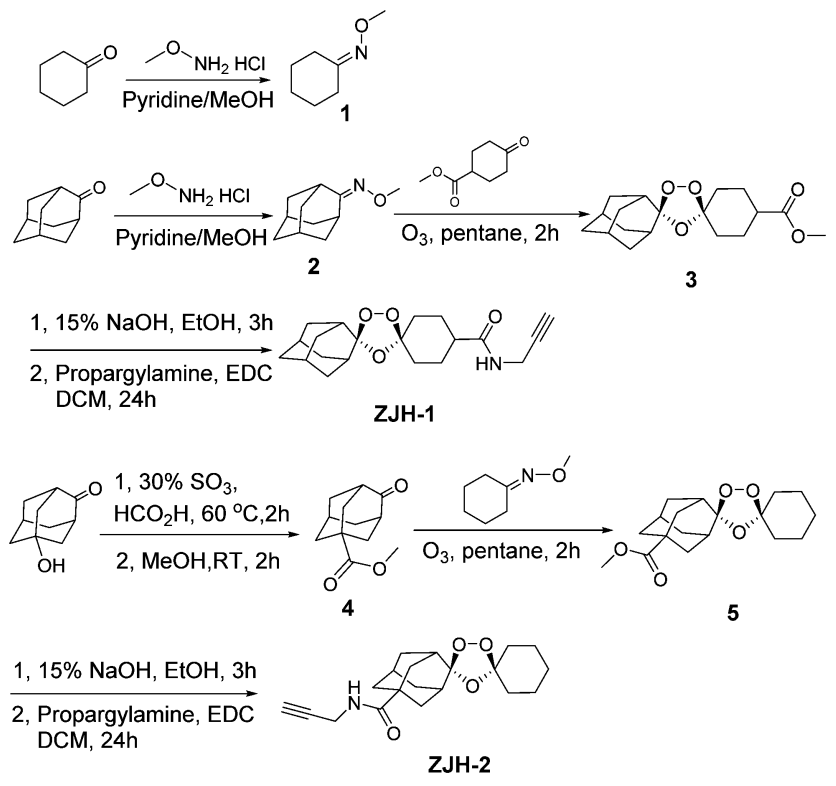

Scheme 1 Synthetic routes of probes $\mathrm{ZJH}-1$ and $\mathrm{ZJH}-2$.
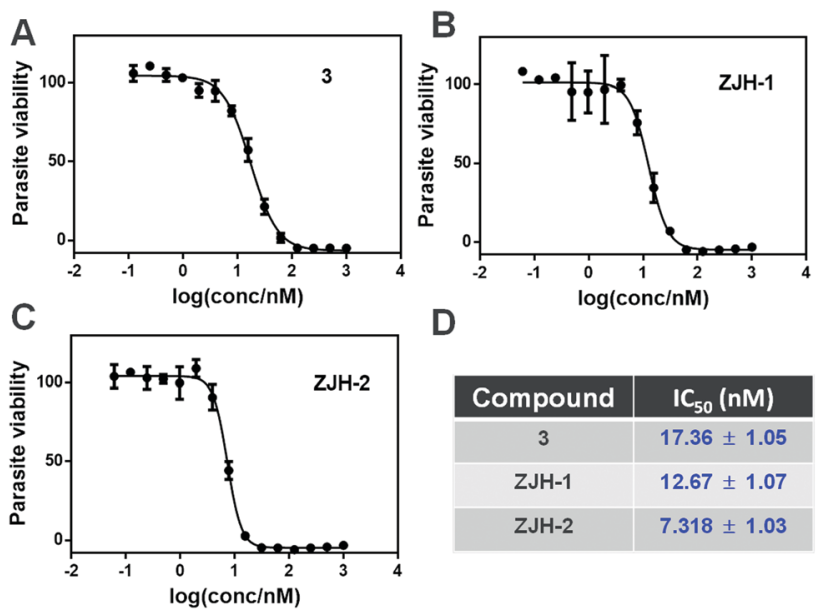

D

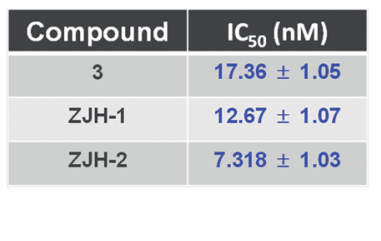

Fig. 2 The viability of malaria parasites treated with 1,2,4-trioxlane-based probes. The viability of malaria parasite was measured in the presence of different concentrations of compound 3 (A), ZJH-1 (B) and $\mathrm{ZJH}-2$ (C). (D) The calculated $I C_{50}$ of the three compounds (mean \pm standard deviation).

and ZJH-2 were confirmed by NMR spectroscopy and highresolution mass spectrometry (ESI, $\dagger$ Fig. S1-S14).

We measured the antimalarial activity of probes $\mathrm{ZJH}-1$ and ZJH-2 against $P$. falciparum 3D7 in vitro. Synchronization of parasite cultures with two rounds of $5 \%(\mathrm{w} / \mathrm{v})$ D-sorbitol was performed as previously described. ${ }^{12}$ Compound 3 was included as a positive control. The parasite sensitivity of compound 3, ZJH-1 and ZJH-2 was measured using SYBR Green I as an indicator. ${ }^{13}$ The excitation and emission wavelengths of SYBR Green I were set as 485 and $528 \mathrm{~nm}$, respectively. All three compounds could effectively inhibit the parasite growth and the calculated $\mathrm{IC}_{50}$ values were $12.67 \mathrm{nM}, 7.32 \mathrm{nM}$ and $17.36 \mathrm{nM}$ for ZJH-1, ZJH-2 and compound 3, respectively (Fig. 2). These results indicate that incorporation of an alkyne in probes $\mathrm{ZJH}-1$ and ZJH-2 did not perturb the antimalarial activity confirming that these probes phenocopy the parent trioxolane scaffold and are thus suitable for ABPP.

To assess the ability of probes ZJH-1 and ZJH-2 to label proteins in malaria parasites, different concentrations of probes $\mathrm{ZJH}-1$ and $\mathrm{ZJH}-2$ ranging from 2 to $20 \mu \mathrm{M}$ were incubated with the mixed stage of $P$. falciparum parasite cultures for 4 hours at $37{ }^{\circ} \mathrm{C}$. Red blood cells were removed by lysis with $0.1 \%$ saponin and parasites were pelleted down by centrifugation. The labeled proteome in the parasites was obtained by sonication and the probe-modified proteins were labeled with a fluorescent dye (rhodamine-azide conjugate) using standard copper-catalyzed click chemistry conditions. The treated proteome was resolved by denaturing gel electrophoresis and visualized by in-gel fluorescence scanning (Fig. 3A and ESI, $\dagger$ Fig. S15). We observed that ZJH-2 efficiently labeled proteins at the lowest probe concentration examined $(2 \mu \mathrm{M})$, whereas $\mathrm{ZJH}-1$ displayed virtually no labeling at this concentration and could only label a few proteins at the highest concentration evaluated $(20 \mu \mathrm{M})$. This result indicates that the secondary-carbon-centered radical predominates protein labeling of synthetic 1,2,4-trioxolanes in malaria parasites. The poor protein labeling of the primary-carbon-centered radical 
A

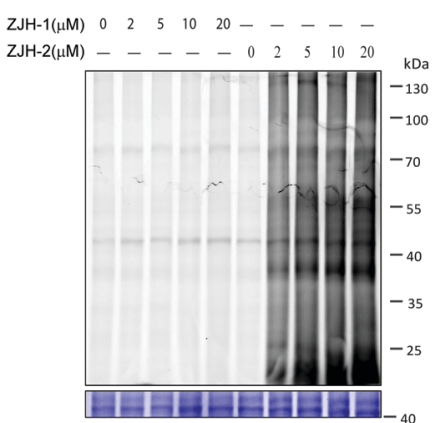

C

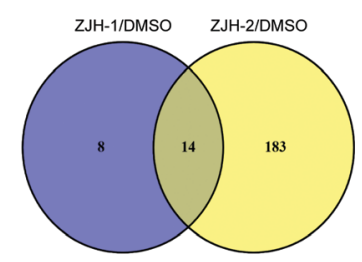

B
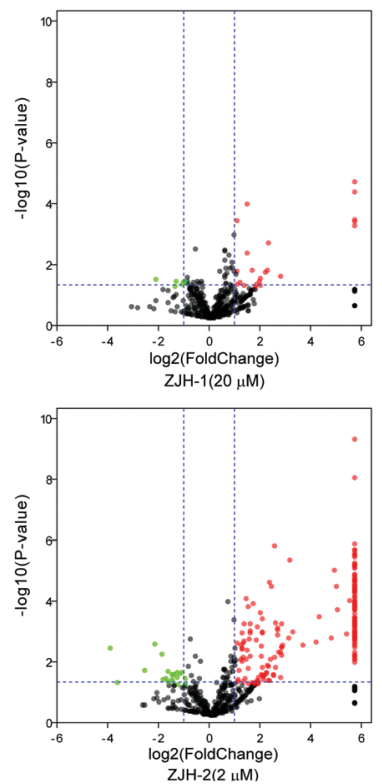

Fig. 3 (A) In situ labelling with different concentrations of probes $\mathrm{ZJH}-1$ or $\mathrm{ZJH}-2$ in malaria parasites. (B) Volcano plots of proteins pulled-down by the probe $\mathrm{ZJH}-1(20 \mu \mathrm{M})$ and $\mathrm{ZJH}-2(2 \mu \mathrm{M})$. The $P$-value was set at 0.05 , and the cut-off value of fold change was 2. (C) Venny analysis of the labeled proteins for $\mathrm{ZJH}-1$ and $\mathrm{ZJH}-2$.

could be ascribed to two causes. First, because of the steric hindrance, the coordination of heme to the endoperoxide likely occurs at the least hindered oxygen atom resulting in generation of the secondary-carbon-centered one. Second, the primarycarbon-centered radical is less stable than the secondary carboncentered one.

To further support the result of gel-based imaging, we next employed gel-free quantitative proteomics to pull down and identify the corresponding protein targets of ZJH-1 and ZJH-2 in parasites. Briefly, the alkyne labeled parasite proteome was reacted with biotin-azide, followed by enrichment with streptavidin beads. Enriched beads were subjected to on-bead digestion with trypsin to release peptide fragments of bound proteins. Collected peptides were separated and detected by LC-MS/MS and the results were processed with the label-free proteomics method to identify the protein targets of ZJH-1 and ZJH-2. During data processing, we used a strict cut-off fold change of 2 and a $p$-value of 0.05 as the qualification criterion. Accordingly the probe $\mathrm{ZJH}-1$ targeted 22 proteins at the concentration of $20 \mu \mathrm{M}$, while $\mathrm{ZJH}-2$ targeted 197 proteins at the concentration of $2 \mu \mathrm{M}$ (Fig. 3B, ESI, $\dagger$ sheet 6 and sheet 7 in proteomics data.xlsx). There were 14 proteins labeled by both ZJH-1 and ZJH-2 (Fig. 3C ànd ESI, $\dagger$ Table S1). These results confirm that the secondary-carbon-centered radical is much more effective in protein-labeling than the primary-carboncentered one. The recent clinical study of OZ439 showed that the drug's peak plasma concentration is around $3 \mu \mathrm{M} .{ }^{14}$ Taken together, our finding suggests that the secondary-carboncentered radical from trioxolane accounts for nearly all the alkylated proteins in vivo.

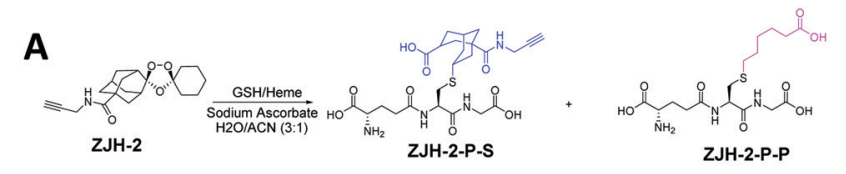

B

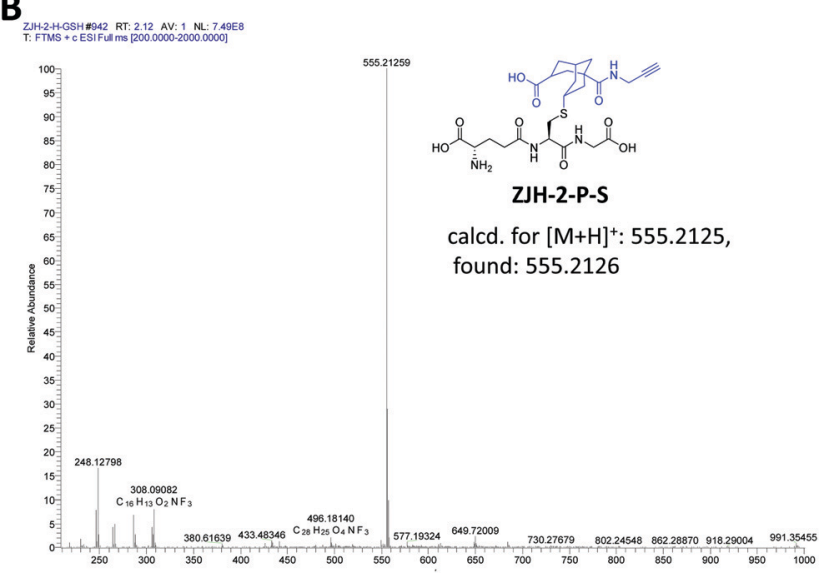

Fig. 4 Reaction between $\mathrm{ZJH}-2$ and $\mathrm{GSH}$ in the presence of heme. (A) Reaction scheme of $\mathrm{ZJH}-2$ and $\mathrm{GSH}$ in the presence of heme and sodium ascorbate; (B) mass spectrum of the main product of $\mathrm{ZJH}-2-\mathrm{P}-\mathrm{S}$.

To further support the result of the chemical proteomics study, we carried out an in vitro experiment to check the products of radical-induced alkylation. Briefly, probes $\mathrm{ZJH}-1$ and $\mathrm{ZJH}-2$ were respectively incubated with hemin and glutathione (GSH) in the presence of sodium ascorbate. We checked the reaction by LC-MS. The expected reaction products between the probes and GSH are shown in Fig. 4A and ESI, $\dagger$ Fig. S17A. In the LC-MS profiles, we found that the main products generated by secondary-carbon-centered radicals (ZJH-1-P-S and ZJH-2-P-S) have a single ion peak in the mass spectra with the expected $m / z$ ratio (Fig. 4B, ESI, $\dagger$ Fig. S16C, D and S18). However, it is difficult to find the ion peak for the product generated by the primary-carbon-centered radicals (ZJH-1-P-P and ZJH-2-P-P) (ESI, $\dagger$ Fig. S16D and S17E). This result also indicated that the secondary-carbon-centered radicals are produced much more than the primary-carbon-centered ones after the trioxolane group was activated by heme.

In conclusion, we designed two molecular probes to investigate the protein labeling capacity of primary and secondary carbon-centered radicals generated by synthetic trioxolanes. Both gel-based and gel-free proteomics methods demonstrated that the secondary-carbon-centered radical was responsible for nearly all the protein labeling in situ. These results provide a more nuanced insight into the unique mode of action of the endoperoxide-containing antimalarials. This study may also aid the further development of potent anti-malaria drugs.

We greatly thank Prof. Courtney Aldrich of the University of Minnesota for careful editing of our manuscript. We also thank the start-up funding from the State Key Laboratory of Bioactive Substances and Functions of Natural Medicines, Institute of Materia Medica, Peking Union Medical College, and CAMS Innovation Fund for Medical Sciences (CIFMS) (2017-I2M-4005, 2016-I2M-1-019). 


\section{Conflicts of interest}

The authors declare that there is no conflict of interest in the publication of this article.

\section{Notes and references}

1 https://www.who.int/malaria/publications/world-malaria-report-2018/ report/en/.

2 T. S. Kaufman and E. A. Rúveda, Angew. Chem., Int. Ed., 2005, 44, 854-885.

3 K. Krafts, E. Hempelmann and A. Skórska-Stania, Parasitol. Res., 2012, 111, 1-6.

4 A. F. G. Slater, Pharmacol. Ther., 1993, 57, 203-235.

5 Y. Tu, Angew. Chem., Int. Ed., 2016, 55, 10210-10226.

6 (a) Z. Guo, Acta Pharm. Sin. B, 2016, 6, 115-124; (b) Y. Wu, Acc. Chem. Res., 2002, 35, 255-259.

7 (a) J. L. Vennerstrom, S. Arbe-Barnes, R. Brun, S. A. Charman, F. C. K. Chiu, J. Chollet, Y. Dong, A. Dorn, D. Hunziker, H. Matile, K. Mclntosh, M. Padmanilayam, J. S. Tomas, C. Scheurer, B. Scorneaux, Y. Tang, H. Urwyler, S. Wittlin and W. N. Charman, Nature, 2004, 430, 900-904; (b) S. A. Charman, S. Arbe-Barnes, I. C. Bathurst, R. Brun, M. Campbell, W. N. Charman, F. C. K. Chiu, J. Chollet, C. Craft, D. J. Creek, Y. Dong, H. Matile, M. Maurer, J. Morizzi, T. Nguyen, P. Papastogiannidis, C. Scheurer, D. M. Shackleford, K. Sriraghavan, L. Stingelin, Y. Tang, H. Urwyler, X. Wang, K. L. White, S. Wittlin, L. Zhou and J. L. Vennerstrom, Proc. Natl. Acad. Sci. U. S. A., 2011, 108, 4400-4405.

8 (a) J. Wang, C.-J. Zhang, W. N. Chia, C. C. Y. Loh, Z. Li, Y. M. Lee, Y. He, L.-X. Yuan, T. K. Lim, M. Liu, C. X. Liew, Y. Q. Lee, J. Zhang, N. Lu, C. T. Lim, Z.-C. Hua, H.-M. Shen, K. S. W. Tan and Q. Lin, Nat. Commun., 2015, 6, 10111; (b) H. M. Ismail, V. Barton, M. Phanchana,
S. Charoensutthivarakul, M. H. L. Wong, J. Hemingway, G. A. Biagini, P. M. O'Neill and S. A. Ward, Proc. Natl. Acad. Sci. U. S. A., 2016, 113, 2080-2085; (c) H. M. Ismail, V. Barton, M. Phanchana, S. Charoensutthivarakul, G. A. Biagini, S. A. Ward and P. M. O'Neill, Angew. Chem., Int. Ed., 2016, 55, 6401-6405; (d) C.-J. Zhang, J. Wang, J. Zhang, Y. M. Lee, G. Feng, T. K. Lim, H.-M. Shen, Q. Lin and B. Liu, Angew. Chem., Int. Ed., 2016, 55, 13770-13774; (e) W. Li, Y. Zhou, G. Tang and Y. Xiao, Bioconjugate Chem., 2016, 27, 2828-2833.

9 Y. Tang, Y. Dong, X. Wang, K. Sriaghavan, J. K. Wood and J. L. Vennerstrom, J. Org. Chem., 2005, 70, 5103-5110.

10 J. Hioe and H. Zipse, Org. Biomol. Chem., 2010, 8, 3609-3617.

11 (a) C. Drahl, B. F. Cravatt and E. J. Sorensen, Angew. Chem., Int. Ed., 2005, 44, 5788-5809; (b) T. F. Gronauer, M. M. Mandl, M. Lakemeyer, M. W. Hackl, M. Meßner, V. S. Korotkov, J. Pachmayr and S. A. Siever, Chem. Commun., 2018, 54, 9833-9836; (c) Z. Li, P. Hao, L. Li, C. Y. J. Tan, X. Cheng, G. Y. J. Chen, S. K. Sze, H.-M. Shen and S. Q. Yao, Angew. Chem., Int. Ed., 2013, 52, 8551-8556; (d) H. Guo, J. Xu, P. Hao, K. Ding and Z. Li, Chem. Commun., 2017, 53, 9620-9623; (e) H. Park, J. Y. Koo, Y. V. Srikanth, S. Oh, J. Lee, J. Park and S. B. Park, Chem. Commun., 2016, 52, 5828-5831; $(f)$ N. Chen, J. Liu, Z. Qiao, Y. Liu, Y. Yang, C. Jiang, X. Wang and C. Wang, Chem. Sci., 2018, 9, 2826-2830.

12 C. Lambros and J. P. Vanderberg, J. Parasitol., 1979, 65, 418-420; S. Pan, H. Zhang, C. Wang, S. C. L. Yao and S. Q. Yao, Nat. Prod. Rep., 2016, 33, 612-620.

13 (a) T. N. Bennett, M. Paguio, B. Gligorijevic, C. Seudieu, A. D. Kosar, E. Davidson and P. D. Roepe, Antimicrob. Agents Chemother., 2004, 48, 1807-1810; (b) M. Smilkstein, N. Sriwilaijaroen, J. X. Kelly, P. Wilairat and M. Riscoe, Antimicrob. Agents Chemother., 2004, 48, 1803-1806.

14 A. P. Phyo, P. Jittamala, F. Hosten, S. Pukrittayakamee, M. Imwong, N. J. White, S. Duparc, F. Macintyre, M. Baker and J. J. Möhrle, Lancet Infect. Dis., 2016, 16, 61-69. 